\title{
751 NEO-X-PRIME BISPECIFIC ANTIBODIES TARGETING CD40 AND TUMOR ANTIGENS PROMOTE CROSS- PRESENTATION OF TUMOR EXOSOME-DERIVED NEOANTIGEN AND INDUCE SUPERIOR ANTI-TUMOR RESPONSES COMPARED TO CD40 MAB
}

${ }^{1}$ Karin Hagerbrand*, ${ }^{1}$ Mattias Levin, ${ }^{1}$ Laura Von Schantz, 'Laura Varas, ${ }^{1}$ Anna Säll,

${ }^{1}$ Adnan Deronic, ${ }^{1}$ Anette Sundstedt, 'Lill Ljung, ${ }^{1}$ Karin Barchan, ${ }^{1}$ Doreen Werchau, ${ }^{1}$ Anna Rosén, 'Barnabas Nyesiga, 'Eva Lindqvist, 'Mia Thagesson, ${ }^{1}$ Peter Ellmark. ${ }^{1}$ Alligator Bioscience, Lund, Sweden; ${ }^{2}$ Alligator Bioscience $A B$, Lund, Sweden

Background Alligator's Neo-X-Prime platform aims to enable antigen presenting cells to efficiently enhance priming of tumor neoantigen-specific $\mathrm{T}$ cells with the goal of overcoming PD-1 resistance in certain tumor types. We hypothesize that binding of a CD40 x TAA bispecific antibody (bsAb) to CD40 on dendritic cells (DCs) and a tumor-associated antigen (TAA) on tumor exosomes or tumor debris leads to (i) activation of the DC, (ii) uptake of the tumor material, (iii) cross-presentation of tumor-derived neoantigen (present in exosomes or debris) and, iv) priming of tumor neoantigen-specific $\mathrm{T}$ cells, resulting in an increased quantity and/or quality of the tumortargeting $\mathrm{T}$ cell pool.

Methods Functionality was evaluated in vitro using CD40 reporter cells and monocyte-derived DCs, co-cultured with cells expressing TAA. Further, co-localization of TAA-expressing cellular debris with a CD40-expressing human B cell line in the presence of bsAbs was assessed using live cell imaging. In vivo, anti-tumor efficacy and immunological memory were assessed in human CD40 transgenic (hCD40tg) mice bearing MB49 bladder carcinoma tumors transfected with human TAA or controls. T cells isolated from OVA-specific TCR-transgenic mice were used to evaluate the effect of Neo-X-Prime bsAbs on antigen-specific $\mathrm{T}$ cell expansion in the presence of hCD40tg DCs and exosomes from MB49 tumors transfected with both human TAA and OVA using flow cytometry.

Results Using CEA as a highly expressed TAA, we have developed lead Neo-X-Prime CD40-CEA bsAbs engineered to achieve an optimal profile. Further, using Neo-X-Prime concept molecules targeting EpCAM, we have demonstrated the ability to mediate co-localization of tumor debris and CD40 expressing antigen presenting cells that is dependent on the receptor density of the TAA. We have further shown that addition of Neo-X-Prime bsAbs to a co-culture of murine DCs, $\mathrm{T}$ cells and tumor-derived exosomes induces increased expansion of model neoantigen-specific $\mathrm{T}$ cells. In vivo, Neo$\mathrm{X}$-Prime bsAbs display a potent, TAA-dependent anti-tumor effect that is superior to CD40 mAbs. Cured mice develop a broad immunological memory that is not dependent on expression of the TAA. The tumor-localizing property of Neo$\mathrm{X}$-Prime bsAbs also shows potential for improved safety compared to CD40 monospecific antibodies.

Conclusions Neo-X-Prime bsAbs have the potential to tumorselectively target CD40-expressing antigen-presenting cells to mediate an expansion of the tumor-specific $\mathrm{T}$ cell repertoire, resulting in increased $\mathrm{T}$ cell infiltration and potent anti-tumor effects.

Ethics Approval All experiments were performed after approval from the Malmö/Lund Animal Ethics Committee.

http://dx.doi.org/10.1136/jitc-2021-SITC2021.751 